\title{
Periampuller divertikül ERCP'de pankreatiti artırır kanamayı arttırmaz
}

\author{
Periampullary diverticula increase post-ERCP pancreatitis, but not bleeding
}

H. Mehmet SÖKMEN, Kamil ÖZDIL, Abdurrahman ŞAHIN, Resul KAHRAMAN, Bilgehan YÜZBAŞIOĞLU, Turan ÇALHAN, Hakan DEMIRDAĞ, Adil NIĞDELIOĞLU

Ümraniye Eğitim ve Araștırma Hastanesi Gastroenterohepatoloji Kliniği, Istanbul

\begin{abstract}
Giriş ve Amaç: Periampuller divertiküllü hastalarda endoskopik retrograd kolanjiopankreatikografi işleminin normal hastalara göre daha zor ve komplikasyon açısından daha riskli olduğu sanılmaktadır. Genelde, endoskopik retrograd kolanjiopankreatikografi yapılan hastalarda periampuller divertikül prevelansı \%5-32 arasında saptanmıștır. Çalıșmamızda, çeşitli nedenlerle endoskopik retrograd kolanjiopankreatikografi endikasyonu konan hastalarımızda periampuller divertikül sıklığı ve periampuller divertikülün endoskopik retrograd kolanjiopankreatikografi yapılan hastalarda komplikasyon oranlarını artırıp arttırmadığının retrospektif değerlendirilmesi amaçlandı. Gereç ve Yöntem: Temmuz 2009 ile Ekim 2010 arasinda ünitemizde yapılan endoskopik retrograd kolanjiopankreatikografi isslemleri retrospektif olarak değerlendirildi. Endoskopik retrograd kolanjiopankreatikografi yapılan hastalar periampuller divertikül bulunup bulunmamasina göre iki gruba ayrıldd. Periampuller divertikül mevcudiyetine göre endoskopik retrograd kolanjiopankreatikografi komplikasyonları değerlendirildi. Bulgular: Çeşitli nedenlerle endoskopik retrograd kolanjiopankreatikografi yapılan 216 hastanın 3l'inde $(\% 14,6)$ periampuller divertikül vard.. Bu hastalara yapılan toplam 258 işlem değerlendirmeye alındı. Periampuller divertikülü olan hastaların ortalama yaşı $(71.4 \pm 12.4$ yıl), periampuller divertikülü olmayan hastalardan $(58.3 \pm 17.3$ yll) yüksekti $(p<0.001)$. Periampuller divertikülü olan grupta kadın oranı, periampuller divertikülü olmayan gruptaki kadın oranından yüksekti (sırasıyla, \%72.4'e karșı \%54.9, p=0.04). Kanama ve perforasyon komplikasyon oranları yönünden gruplar arsı anlamlı fark yoktu. Ancak, post-endoskopik retrograd kolanjiopankreatikografi pankreatit periampuller divertikül grubunda (\%18), periampuller divertiküllü olmayan gruba (\%6,8) göre daha yüksekti $(p=0,02)$. Sonuç: Periampuller divertikül, özellikle yașlllarda ve kadınlarda sık görülür. Komplikasyon olarak kanama ve perforasyonu artırmaz. Bu hastalarda endoskopik retrograd kolanjiopankreatikografinin majör komplikasyonu post-endoskopik retrograd kolanjiopankreatikografi pankreatittir. Periampuller divertikül hastalarmndaki bu komplikasyon divertikül varlığı nedeniyle değişen papilla pozisyonu nedeniyle olabilir.
\end{abstract}

Anahtar kelimeler: Periampuller divertikül, endoskopik retrograd kolanjiopankreatikografi, komplikasyon

\section{GİRIS}

Periampuller divertikül (PAD), duodenum ikinci kısmı medial tarafta bulunan, içerisinde veya kenarında Ampulla Vateri'nin yerleştiği kazanılmış bir luminal anomalidir (Şekil 1). Yapılan radyolojik çalışmalarda ve postmortem serilerde prevalansı yaklaşı $\% 5$ olarak bildirilmektedir (1). Çoğunlukla asemptomatik olup, daha çok 40 yaşından sonra görülmektedir. Ancak, endoskopik retrograd kolanjiopankreatikografi (ERCP) yapılan hastalarda sıklığı \%5-32 oranında değişmek-
Background/aims: It is suggested that the endoscopic retrograde cholangiopancreatography procedure is more difficult and carriers higher risk of complication in patients with periampullary diverticula than in normal subjects. Prevalence of periampullary diverticula is 5\% to $32 \%$ in patients who undergo endoscopic retrograde cholangiopancreatography. In this study, we aimed to investigate the prevalence of periampullary diverticula in patients who underwent endoscopic retrograde cholangiopancreatography for various indications and the impact of periampullary diverticula on the complications of endoscopic retrograde cholangiopancreatography. Materials and Methods: Consecutive endoscopic retrograde cholangiopancreatography procedures in our endoscopy unit performed between July 2009 and October 2010 were evaluated retrospectively. Patients who underwent an endoscopic retrograde cholangiopancreatography were divided into two groups according to the presence or not of periampullary diverticula. The complications were evaluated according to the presence of periampullary diverticula. Results: Periampullary diverticula were present in 31 (14.6\%) of the $213 \mathrm{pa}-$ tients undergoing endoscopic retrograde cholangiopancreatography for various indications. A total of 258 procedures were evaluated. The mean age was significantly higher in patients with periampullary diverticula ( $71.4 \pm 12.4$ years) than in the control group $(58.3 \pm 17.3$ years $)(p<0.001)$. The proportion of females was greater in the group with periampullary diverticula than in the group without periampullary diverticula $(74.2 \%$ vs $54.9 \%$, respectively, $p=0.04$ ). There was no significant difference between the groups in the complication rate of bleeding and perforation; however, post- endoscopic retrograde cholangiopancreatography pancreatitis was higher in patients with periampullary diverticula (18\%) than in those without periampullary diverticula (6.8\%) ( $p=0.02)$. Conclusions: Periampullary diverticula are common, especially in older and female patients, and do not significantly increase some complications such as bleeding and perforation. Post- endoscopic retrograde cholangiopancreatography pancreatitis is the major complication of endoscopic retrograde cholangiopancreatography in patients with periampullary diverticula. This complication in patients with periampullary diverticula might be due to the position of the papilla, which can be modified by the presence of the diverticulum.

Key words: Periampullary diverticula, endoscopic retrograde cholangiopancreatography, complication

tedir (Şekil 2) (1, 2). ERCP işlemi ile ilişkili olan en sık komplikasyonlar; kanama, perforasyon ve pankreatit olarak siralanabilir. Bunun yanında, güç kanülasyon da morbidite artışına katkıda bulunan bir diğer faktördür. PAD bulunan hastalarda komplikasyon sıklığında ve başarısız kanülasyonda artış olduğunu gösteren yayınlar mevcuttur (3-5). Bu çalışmada ERCP yaptığımız olgularda PAD sıklığını ve komplikasyonları araştırmayı amaçladık. 


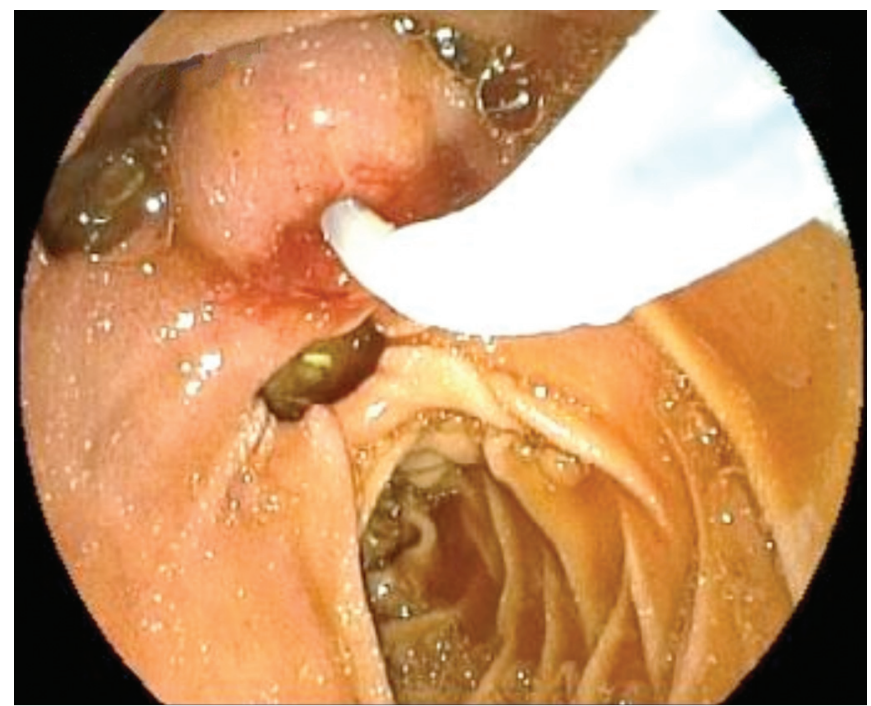

Şekil 1. Bir hastada periampüller divertikülün endoskopik görünümü

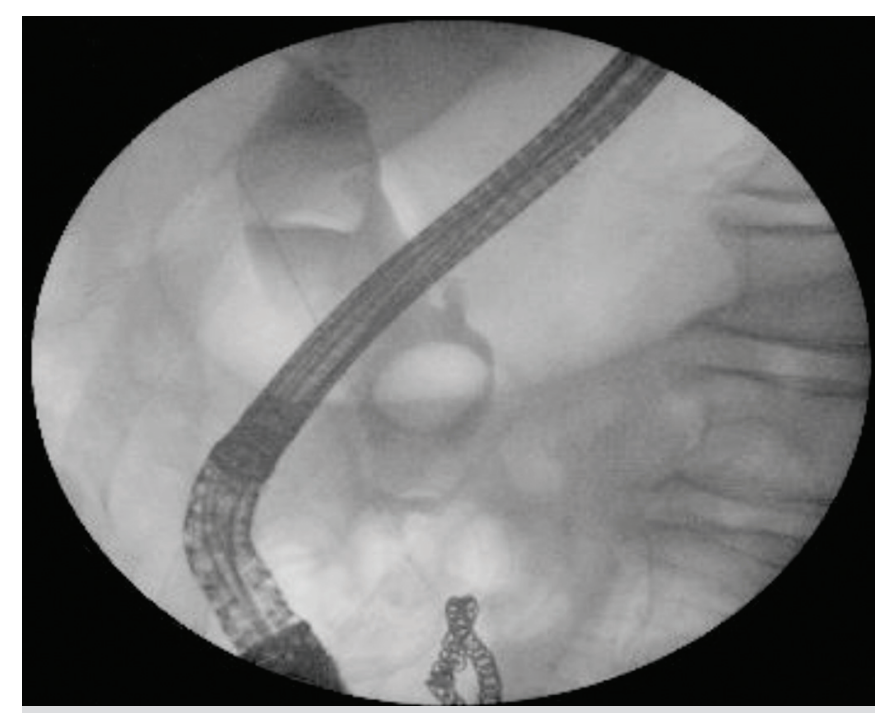

Şekil 2. Aynı hastada periampüller divertikülün radyolojik görüntüsü

\section{GEREC VE YÖNTEMLER}

Ümraniye Eğitim ve Araştırma Hastanesi Gastroenteroloji Kliniği Endoskopi Ünitesi’nde Temmuz 2009 ile Ekim 2010 tarihleri arasında ERCP yapilan ve yeterli verileri olan hastaların dosyaları retrospektif olarak incelendi. Hastalar PAD olan ve PAD olmayan olarak iki gruba ayrıldı. Birden fazla seans işlem yapılan hastalarda demografik özellikler ve tanı yönünden değerlendirmede hasta sayısı, stent uygulanması, ERCP komplikasyonları ve mortalite yönünden işlem sayısına göre gruplar karşılaştırıldı. Çalışma verileri değerlendirilirken tanımlayıcı istatistiksel metodların (Ortalama, standart sapma, frekans) yanısıra verilerin karşılaştırılmasında student $\mathrm{t}$ testi ve niteliksel verilerin karşılaştırılmasında Ki-Kare ve Fishers Exact Ki-kare testi kullanıldı.

\section{BULGULAR}

Toplam 213 hastaya (Kadın/erkek: 123/90) yapilan 258 ERCP işlemi değerlendirildi. PAD 31 (\%14,6) hastada bulundu. PAD'e sahip olan grupta ortalama yaş, PAD olmayan gruba göre daha yüksekti (sırasıyla 71,4 412,4 yıla karşı, $58,3 \pm 17,3$ yll: $\mathrm{p}<0,001$ ). PAD olan grupta kadın oranı, PAD olmayan gruba göre daha yüksekti (sırasıyla \%74,2'ye karşı, \%54,9: p=0,04) (Tablo 1 ve Şekil 3). En sık ERCP tanı ve endikasyonu sırasıyla, koledokolitiazis (\%70) ve biliyer pankreatit $(\% 7,8)$ şeklindeydi. PAD grubunda 30 hastaya $(\% 96,7)$, PAD olmayan grupta ise $134(\% 73,6)$ hastaya safra taşı ve ilişkili klinik durumlar nedeniyle ERCP işlemi yapıldı. PAD olan grupta malignite görülmezken, PAD olmayan grupta 21 hastaya $(\% 11,4)$ malignite nedeniyle işlem uyguland. Gruplara göre ERCP tanıları Tablo l'de verilmiştir. PAD grubunda 5 hastada, PAD olmayan grupta 29 hastada 2 veya üzeri seans uygulandi. Tekrarlayan seans nedenleri, kanüle edilememekten ziyade, çok büyük ve çok sayıda taş olması, kolanjit varlığı ve stent değissimi olarak sıralanabilir. PAD olmayan grupta 6 hasta $(\% 3,3)$, PAD grubunda ise 3 hasta $(\% 9,7)$ kanüle edilemedi ( $>>0,05)$. ERCP işlemi yapılan 33 hastada toplam 48 isslemde, PAD grubunda 7 (\%18,4), PAD olmayan grupta $41(\% 18,6)$ işlemde stent yerleştirme ve değişimi uygulandı ( $>0,05)$. Onaltı hastada (\%48) stent uygulanma nedeni maligniteydi. Kanama PAD olan grupta hiç görülmezken, PAD olmayan grupta $18(\% 7)$ hastada görüldü ( $\mathrm{p}=0,07)$. Sadece 2 hastada transfüzyon ve skleroterapi gerektiren kanama görüldü. ERCP sonrası pankreatit gelişme oranı ise PAD'e sahip grupta, PAD olmayan grupa göre daha yüksekti (sırasıyla \%18,4'e karşı, \%6,8: $\mathrm{p}=0,02$ ). Perforasyon PAD olmayan grupta 2 hastada görüldü. Bu hastalardan birisi koledokolitiazis tanısıyla ERCP yapılan 71 yaşında bir hastaydı. Konservatif izlemle hasta klinik olarak düzeldi. Primer sklerozan kolanjit tanısıyla ERCP yapılan 64 yaşındaki diğer hasta ise stent yerleştirme sirasında olan perforasyon nedeniyle opere edildi. PAD olan grupta perforasyon gözlenmedi. İşlem esnasında ve sonrasında kardiopulmoner komplikasyon gelişimi divertikülsüz grupta 5 hastada görüldü. Bu hastalardan 2'sinde işlem esnasında bradikardi saptandı. Diğer iki hastada ise, işlemin sonlandırılmasını gerektiren hipoksi gelişimi mevcuttu. Mortalite yönünden değerlendirildiğinde, PAD olmayan grupta 2 hasta kaybedildi. Doksanbeş yaşındaki bir hastada işlemden sonra myokard infarktüsü gelişimine bağlı ölüm gözlenirken, diğer hasta işlemden 7 gün sonra sepsis ve buna bağlı çoklu organ yetmezliği nedeniyle öldü. Gruplara göre komplikasyonlar Tablo 2 ve Şekil 4'de verilmiştir.

\section{TARTIȘMA}

Duodenum, divertiküllerin kolondan sonra en sık ikinci yerleşim yeridir. Çoğu asemptomatik olup radyolojik ve endoskopik tetkikler esnasında saptanmasına karşın, divertiküllerin 


\begin{tabular}{|c|c|c|c|}
\hline Tanı & PAD olmayan $n(\%)$ & PAD olan $n(\%)$ & Toplam n (\%) \\
\hline Yaş* & $58,3 \pm 17,3$ & $71,4 \pm 12,4$ & $<0,001 \dagger$ \\
\hline Cinsiyet (kadın) n (\%) & $100(54,9)$ & $23(74,2)$ & $0,04 \dagger$ \\
\hline Koledokolitiyazis & $115(63,2)$ & $29(93,5)$ & $144(67,6)$ \\
\hline Biliyer pankreatit & $19(10,4)$ & $1(3,2)$ & $20(9,4)$ \\
\hline Koledok darlığı & $10(5,5)$ & $1(3,2)$ & $11(5,2)$ \\
\hline Kolanjioselüler karsinom & $7(3,8)$ & $0(0)$ & $7(3,3)$ \\
\hline Pankreas karsinomu & $7(3,8)$ & $0(0)$ & $7(3,3)$ \\
\hline Periampüller bölge tümörü & $6(3,3)$ & $0(0)$ & $6(2,8)$ \\
\hline Hepatoselüler karsinom & $1(0,5)$ & $0(0)$ & $1(0,5)$ \\
\hline Oddi sfinkter disfonksiyonu & $8(4,4)$ & $0(0)$ & $8(3,8)$ \\
\hline Kist hidatik & $2(1,1)$ & $0(0)$ & $2(0,9)$ \\
\hline Biliyer siroz & $2(1,1)$ & $0(0)$ & $2(0,9)$ \\
\hline Primer sklerozan kolanjit & $2(1,1)$ & $0(0)$ & $2(0,9)$ \\
\hline Mirizzi sendromu & $2(1,1)$ & $0(0)$ & $2(0,9)$ \\
\hline Koledokobulber fistül & $1(0,5)$ & $0(0)$ & $1(0,5)$ \\
\hline
\end{tabular}

${ }^{*}$ ) Ortalama \pm Standart sapma, $(\dagger)$ p değeri, PAD: Periampüller divertikül

bir kısmı koledok ile ilişkili klinik durumlar veya divertikül inflamasyonu ile ortaya çıkmaktadır. Duodenumda divertiküllerin en sık görüldügü yer Ampulla Vaterinin 2,5 cm çevresidir. Ampullanın divertikülün içerisinde olduğu divertiküller jukstapapiller, ampullanın divertikülün kenarına açıldığı divertiküller parapapiller divertikül olarak adlandırılmaktadır. Bilgisayarlı tomografi ve magnetik rezonans görüntülemeleri duodenal divertikül tanısında ve pankreas başındaki kistik lezyonlardan ayrımında yeterli olmayabilir (6-8). Bu nedenle duodenoskopi PAD tespitinde en hassas tetkiktir. Semptomlar, divertikül büyüklügü ile ilişkilidir. Küçük divertiküllü hastalarda Ampulla Vateri fonksiyonlarında daha az bozulma ve koledokta daha az basınç artışı görülür, ancak koledok alt ucu dar ise koledokta basınç artışı nedeniyle inflamasyona neden olabilir. Büyük divertiküllerde koledoktaki basınç artışı sarılık, kolanjit ve koledokta taş oluşumuna yol açabilir $(9,10)$.

ERCP yapilan hasta serimizde PAD bulunma oranı \% 14,6 olarak bulunmuştur. Bu oran daha önce yapılan çalışmalarda bulunan sonuçlarla uyumludur $(1,2)$. PAD sıklığı yaşla beraber $\operatorname{artar}(2,10,11)$. Çalışmamızda PAD olan grubun yaş ortalaması, olmayan gruba göre daha yüksekti. Daha önce yapılan çalışmalarda PAD bulunmasına göre cinsiyet yönünden kadın predominansı ile ilgili çelişkili sonuçlar bulunmaktadır (2, $11,12)$. Sonuçlarımız PAD'ün kadınlarda daha sık görüldügünü desteklemektedir. Koledok taşları sıklıkla safra kesesinde oluşan taşa sekonder oluşur. Ancak daha nadir olarak enfeksiyon ve staza bağlı olarak primer koledok taşları da görülebilir (13). Koledok taşlarının çoğunun safra taşı ile ilişkili olduğu göz önüne alındığında kadınlarda sık görülmesi beklenir. PAD olmayan hasta grubunda kadın/erkek oranının yaklaşık eşit olmasına karşın PAD olan grupta kadın oranının yüksek olması; PAD olan grupta safra taşı ve ilişkili kompli- kasyonların daha sık izlenebileceğini dolaylı olarak göstermektedir. Çalışmamızda, PAD olmayan gruptaki hastaların yaklaşık \% 75'inde ERCP yapılma nedeni safra taşı ve ilişkili komplikasyonlar iken, PAD grubundaki hastaların tamamına yakınına koledok taşı ve ilişkili komplikasyonlara bağlı ERCP yapılması bu durumu desteklemektedir.

ERCP işlemi sırasında ve sonrasında pankreatit (\%1,3-\%6,7), kanama (\%0,7-\%2), perforasyon (\%0,5-\%5) gibi komplikasyonlar gelişmektedir (13). PAD olan hastalarda yapılan çalışmalarda kanama yönünden çelişkili sonuçlar elde edilmesine karşın, pankreatit ve perforasyon yönünden PAD olmayanlara göre fark olmadığı gösterilmiştir $(5,14)$. Çok merkezli 2347 hastanın katıldığı bir çalışmada divertikül ile kanama arasında tek değişkenli analizde ilişki gösterilmesine karşın,

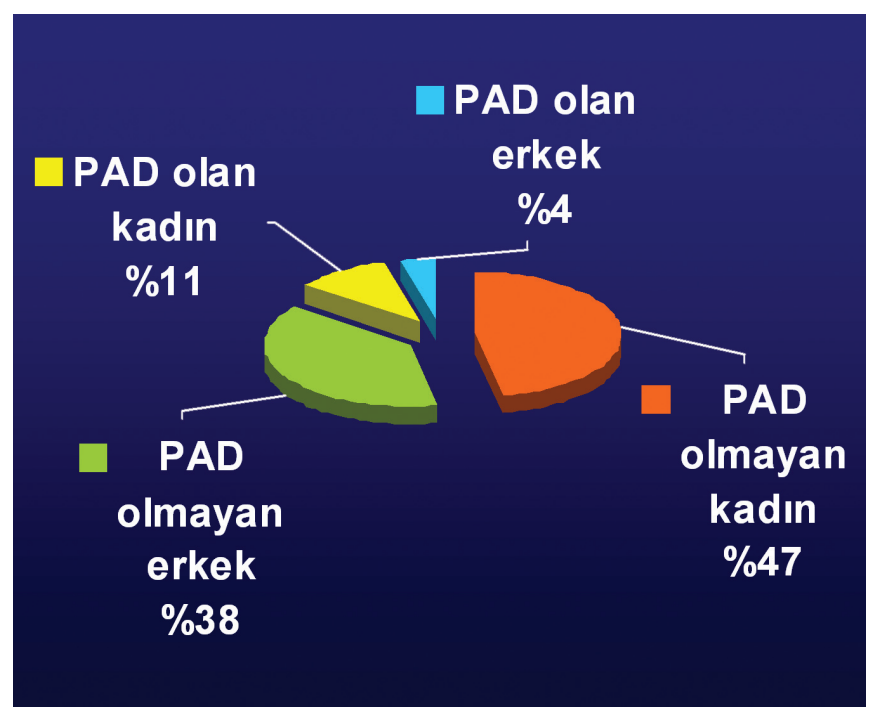

Şekil 3. Divertikül bulunma durumu ve cinsiyete göre hastaların dağılımı PAD: Periampuller divertikül 


\begin{tabular}{|c|c|c|c|c|}
\hline & PAD olmayan & PAD olan & Toplam & $P$ değeri \\
\hline Kanama n (\%) & $\begin{array}{c}18(8,2) \\
(7.0)\end{array}$ & $0(0)$ & $18(7,0)$ & $>0,05$ \\
\hline Perforasyon n (\%) & $\begin{array}{c}2(0,9) \\
(0,8)\end{array}$ & $0(0)$ & $2(0,8)$ & $>0,05$ \\
\hline Pankreatit n (\%) & $\begin{array}{c}15(6,8) \\
(5,8)\end{array}$ & $\begin{array}{c}7(18,4) \\
(2,7)\end{array}$ & $22(8,5)$ & 0,02 \\
\hline Kardiyopulmoner n (\%) & $\begin{array}{c}5(2,3) \\
(1,9)\end{array}$ & $0(0)$ & $5(1,9)$ & $>0,05$ \\
\hline Mortalite n (\%) & $2(0,9)$ & $0(0)$ & $2(0,8)$ & $>0,05$ \\
\hline
\end{tabular}

PAD: Periampüller divertikül

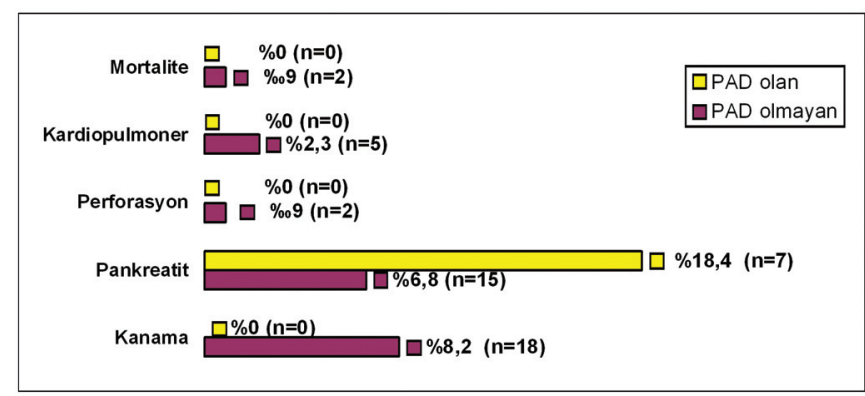

Şekil 4. Divertikül bulunma durumuna göre komplikasyon oranları

çok değişkenli analizde anlamlı ilişki bulunamamıştır. Ayrıca, bir diğer çok merkezli çalışmada kanama ile divertikül bulunması arasında ilişki gösterilememiştir $(15,16)$. Çalışmamızda post-ERCP pankreatit dışındaki diğer komplikasyonlar yönünden, PAD bulunma durumuna göre fark olmadığı görüldü. Divertikül nedeniyle papilla pozisyonunun değişmesi sonucu koledok ve pankreatik kanalda meydana gelen anatomik pozisyonel değişiklikler nedeniyle işlem esnasında pankreatik kanülasyonda artış, pankreatit gelişimine katkıda bulunan önemli bir faktör olabilir. Papilla pozisyonunun değişmesi sonucu ortaya çıkan kanülasyon başarısızlığı da PAD’ün

\section{KAYNAKLAR}

1. Kirk AP, Summerfield JA. Incidence and significance of juxtapapillary diverticula at endoscopic retrograde cholangiopancreatography. Digestion 1980; 20: 31-5.

2. Panteris V, Vezakis A, Filippou G, et al. Influence of juxtapapillary diverticula on the success or difficulty of cannulation and complication rate Gastrointest Endosc 2008; 68: 903-10.

3. Chang-Chien CS. Do juxtapapillary diverticula of the duodenum interfere with cannulation at endoscopic retrograde cholangiopancreatography? A prospective study. Gastrointest Endosc 1987; 33: 298-300.

4. Boix J, Lorenzo-Zúñiga V, Añaños F, et al. Impact of periampullary duodenal diverticula at endoscopic retrograde cholangiopancreatography: a proposed classification of periampullary duodenal diverticula. Surg Laparosc Endosc Percutan Tech 2006; 16: 208-11.

5. Zoepf T, Zoepf DS, Arnold JC, et al. The relationship between juxtapapillary duodenal diverticula and disorders of the biliopancreatic system: analysis of 350 patients. Gastrointest Endosc 2001; 54: 56-61. neden olduğu bir diğer morbidite olarak kabul edilmektedir, ancak bu konudaki veriler çelişkilidir. Tyagi ve arkadaşları, divertikül boyutu ve papillanın yerleşiminin kanülasyon başarısı üzerine etkisi olmadığını göstemişlerdir (11). Ayrıca, Boix ve arkadaşları kanülasyon güçlüğünü 4 gruba ayırarak inceledikleri çalışmada, divertikül olan ve olmayan gruplar arasında kanülasyon dereceleri yönünden divertikül varlığına göre gruplar arasında farklılık olmadığını göstermişlerdir (4). Çalışmamızda, divertikül varlığına bağlı kanülasyon başarısızlığı yönünden gruplar arası farklılık gözlenmemesine karşın, PAD olanlarda olmayanlara göre yaklaşık 3 kat daha fazla bulundu. Çalışmamızın bir kısıtllılı̆ı olarak, çalışmanın retrospektif doğası nedeniyle divertikül boyutu ve yerleşimini değerlendirilemedik.

Bu çalışmada, kanama yönünden gruplar arası istatiksel fark olmamasına karşın PAD olmayan grupta 18 kanama gözlenirken PAD grubunda hiç kanama olmaması, divertiküllü hastalarda kesi sırasında daha dikkatli olunması ile ilişkili olabilir. Ayrıca PAD olmayan hastalarda iğne sfinkterotomla pre-cut papillotominin daha sık kullanılması, bu grup hastalarda kanama komplikasyonunun daha sık görülmesine katkıda bulunan bir diğer faktör olabilir. ERCP sonrası gelişebilecek önemli komplikasyonlardan birisi de kardiyopulmoner yetmezliktir. Başta kardiyopulmoner yetmezlik olmak üzere diğer komplikasyonlar \%0,5-\%2,3 arasında görülmektedir. Bizim çalışmamızda PAD olmayan grupta 5 hastada işlem sonlandırılmasını gerektiren kardiyopulmoner komplikasyonlar gözlendi. Özellikle, yaşlı hastalarda işlem öncesi gerekli kardiopulmoner değerlendirmenin yapılması, işlem sırasında ve sonrasında yakın izlem ile kardiyopulmoner komplikasyonların erken tanı ve tedavisi ile morbidite ve mortalitenin azaltılması sağlanabilir. Serimizdeki hasta sayısının düşük olması nedeniyle mevcut sonuçlar elde edilmiş olabilir. PAD hastalarında komplikasyonların belirlenmesi ve ERCP işleminin başarısı üzerine olan etkilerinin anlaşılabilmesi için daha fazla hasta sayısı ile yapılacak çalışmalara ihtiyaç vardır.
6. Macari M, Lazarus D, Israel G, Megibow A. Duodenal diverticula mimicking cystic neoplasms of the pancreas: CT and MR imaging findings in seven patients. AJR Am J Roentgenol 2003; 180: 195-9.

7. Tsitouridis I, Emmanouilidou M, Goutsaridou F, et al. MR cholangiography in the evaluation of patients with duodenal periampullary diverticulum. Eur J Radiol 2003; 47: 154-60.

8. Kim MH, Myung SJ, Kim YS, et al. Routine biliary sphincterotomy may not be indispensable for endoscopic pancreatic sphincterotomy. Endoscopy 1998; 30: 697-701

9. Kim MH, Myung SJ, Seo DW, et al. Association of periampullary diverticula with primary choledocholithiasis but not with secondary choledocholithiasis. Endoscopy 1998; 30: 601-4

10. Lobo DN, Balfour TW, Iftikhar SY. Periampullary diverticula: consequences of failed ERCP. Ann R Coll Surg Engl 1998; 80: 326-31. 
11. Tyagi P, Sharma P, Sharma BC, Puri AS. Periampullary diverticula and technical success of endoscopic retrograde cholangiopancreatography. Surg Endosc 2009; 23: 1342-5.

12. Leivonen MK, Halttunen JA, Kivilaakso EO. Duodenal diverticulum at endoscopic retrograde cholangiopancreatography, analysis of 123 patients. Hepatogastroenterology 1996; 43): 961-6.

13. Williams EJ, Green J, Beckingham I, et al. Guidelines on the management of common bile duct stones (CBDS). Gut 2008; 57: 1004-21.
14. Tham TC, Kelly M. Association of periampullary duodenal diverticula with bile duct stones and with technical success of endoscopic retrograde cholangiopancreatography. Endoscopy 2004; 36: 1050-3.

15. Shemesh E, Klein E, Czerniak A, et al. Endoscopic sphincterotomy in patients with gallbladder in situ: the influence of periampullary duodenal diverticula. Surgery 1990; 107: 163-6.

16. Loperfido S, Angelini G, Benedetti G, et al. Major early complications from diagnostic and therapeutic ERCP: a prospective multicenter study. Gastrointest Endosc 1998; 48: 1-10. 